ing, one of the most fundamental questions associated with crack dynamics is the maximum speed that cracks can propagate. Depending on the type of loading (e.g., tensile, shear, or antiplane shear), there is a unique maximum speed cracks can achieve. For tensile-loaded cracks, theory predicts that this limiting speed is the Rayleigh wave speed, the speed of elastic waves on a surface. Recent theoretical work, including atomistic simulations, has challenged this classical view. Now, P.J. Petersan and co-workers from the University of Texas at Austin have shown experimentally that tensileloaded cracks in rubber can actually propagate faster than the Rayleigh wave speed and even break the sound barrier.

As reported in the July issue of Physical Review Letters (105504), Petersan and colleagues identified the intersonic crack speed by the observation of shock fronts near the crack tip by high-speed photography (see Figure 1). The experiments were conducted using highly stretched sheets of rubber. In this nonlinear material, cracks in tension (mode I) exceeded the shear wave speed and traveled in the intersonic range between shear and longitudinal wave speeds. These results have important implications for understanding fundamental crack dynamics, demonstrating that the classical understanding of crack dynamics needs to be revised.

What is the physical explanation for this phenomenon? Through observations made earlier in large-scale molecular dynamics simulations, M.J. Buehler and H. Gao of Max Planck Institute, Stuttgart, and F.F. Abraham of Almaden Research Center in San Jose discovered that hyperelasticity (i.e., the elasticity of materials at large strains), although mostly neglected in existing theories of fracture, is crucial in understanding crack dynamics. In their article published in the November 13, 2003, issue of Nature (p. 141), Buehler and colleagues hypothesized that energy flow toward a crack tip occurs in a region whose size is described by a so-called characteristic energy length scale $\chi$. This length scale competes with the size of the hyperelastic region. If the size of the region of energy flow $\chi$ is comparable to the size of the hyperelastic region $\left(r_{\mathrm{H}}\right)$, energy flow is completely dominated by the local largestrain or hyperelastic properties.

"For instance," Buehler said, "if material stiffens with strain as in the case of rubber used in Petersan's experiments and in the molecular dynamics simulations, energy flow is enhanced because of the stiffer material properties, and cracks can thus break through the sound barrier. The observation of intersonic mode I

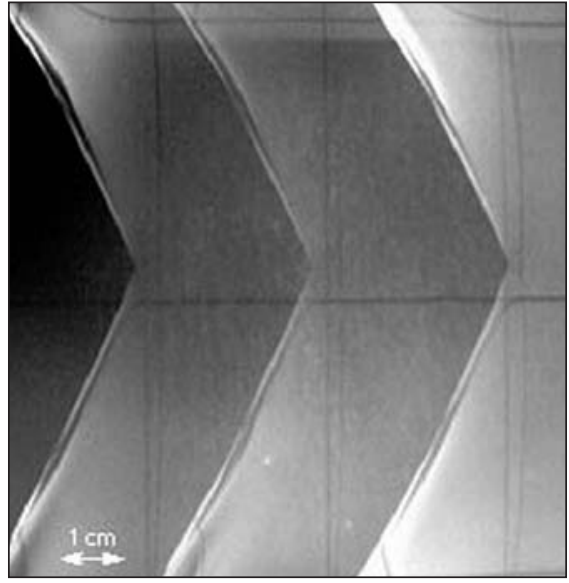

Figure 1. Multiple-exposure photograph of a crack propagating in a rubber sample $\left(\lambda_{x}=1.2, \lambda_{y}=2.4\right)$; speed of the crack, $\sim 56 \mathrm{~m} / \mathrm{s}$

cracks in rubber seems to be an example for the importance of hyperelastic effects in real materials. "

Petersan said, "We agree that the rupture of rubber opens up a new regime in the study of fracture and look forward to understanding the mechanism which explains it."

\section{Fluorine-Containing Molecules Serve as Structure-Directing Agents in Synthesis of Molecular Sieves}

Water-soluble organic molecules have been used as structure-directing agents (SDAs) in the synthesis of zeolites. By filling the cavities in the material, these molecules direct the hydrothermal crystallization of zeolite and zeolite-like materials. Usually, hydrogen atoms attached to the organic molecules are in charge of the chemical interaction with the zeolite inorganic framework. By replacing hydrogen atoms with fluorine atoms as SDAs, J. Pérez-Pariente and colleagues at the Instituto de Catálisis y Petroleoquímica, Spain, significantly changed the surface properties of their zeolites.

As reported in the August 24 issue of Chemistry of Materials (p. 3209), the researchers prepared a group of fluorinecontaining SDAs such as 1-(fluorobenzyl)-pyrrolidine (F-PB) and difluorinated dibenzyldimethyl-ammonium cations (DBDM) by reacting $o_{-}^{-}, m^{-}$, or $p$-fluorobenzyl chloride with pyrrolidine, dimethylamine, or benzyldimethylamine. Inorganic gels and SDAs (1:1:1: $x: 40$ $\mathrm{R}: \mathrm{Al}_{2} \mathrm{O}_{3}: \mathrm{P}_{2} \mathrm{O}_{5}: \mathrm{SiO}_{2}: \mathrm{H}_{2} \mathrm{O}$, where $\mathrm{R}$ is the SDA and $x$ is 0 for alumino-phosphate, $\mathrm{AlPO}$, and 0.5 for silico-alumino-phosphate, SAPO, gels) were heated at $150^{\circ} \mathrm{C}$ for $72 \mathrm{~h}$. Another silica gel (0.54:0.54:1:7.93 $\left.\mathrm{R}: \mathrm{HF}: \mathrm{SiO}_{2}: \mathrm{H}_{2} \mathrm{O}\right)$ with the quaternary monofluorinated DBDM cations was heated at $135^{\circ} \mathrm{C}$ for 46 days in a fluoride medium. The control material AlPO-5 (AFI), which is an aluminophosphate with 12MR (12-membered ring) one-dimensional channels, was made from benzylpyrrolidine $(\mathrm{BP})$.

X-ray diffraction patterns of the different materials revealed that the nature and crystallinity of the monofluorinated derivatives used as SDAs depended strongly on the position of the fluorine atom in the aromatic ring. The crystallinity of the materials was found to be higher when fluorine was in the meta-fluoro $(\mathrm{mF})$ position rather than in the para-fluoro $(\mathrm{pF})$ position, and the stability of the AlPO-5 crystal (framework code type AFI) structure with different SDAs follows the order mF-BP $>\mathrm{BP}>$ oF[ortho]$\mathrm{BP}>>\mathrm{pF}-\mathrm{BP}$. The researchers said that the meta-fluoro derivative served as the best template because of the electrostatic interactions between the fluorine atom and the inorganic zeolite pore; the orthoand para-fluoro derivatives showed poor crystallinity of the AFI phase in the product due to the structural hindrance. Magic angle spinning nuclear magnetic resonance data showed that the template molecules were intact inside both zeolites (DBDM), which were made in a fluoride medium, and $\mathrm{AFI}(\mathrm{BP})$ materials.

LuCY YuE Hu

\section{Flame-Spraying Technique Yields Aluminate Bulk Glasses and Nanoceramics}

Alumina, $\mathrm{Al}_{2} \mathrm{O}_{3}$, is the basis of a number of important ceramic systems. It is desirable to form alumina-rich bulk glasses because of their superior mechanical, chemical, and optical properties. However, it is very difficult to form bulk glass from pure alumina liquid, a reluctant glass former, because of the extremely high cooling rates required, typically in the range of $10^{7} \mathrm{~K} \mathrm{~s}^{-1}$. The addition of rareearth oxides reduces the cooling rates to under $10^{3} \mathrm{~K} \mathrm{~s}^{-1}$, which is still too high to obtain glasses with dimensions of more than a few millimeters. A. Rosenflanz and co-workers at $3 \mathrm{M}$ in Minnesota have utilized a flame-spraying technique to generate glass beads that contain $80 \mathrm{~mol} \%$ alumina (the balance is rare-earth oxide). They then sintered the beads, yielding bulk alumina glass with the appropriate shape. Devitrification of the bulk glass using heat treatment resulted in a nanoceramic with twice the hardness of the original bulk glass.

As Rosenflanz and colleagues reported 